\title{
Supramolecular Alternating Donor-Acceptor Assembly toward Intercalated Covalent Organic Frameworks
}

\author{
Huiqing Li, ${ }^{\dagger}$ Pengpeng Shao, ${ }^{\ddagger}$ Shuqi Chen, ${ }^{\dagger}$ Guosheng Li, ${ }^{\#}$ Xiao Feng, ${ }^{\ddagger}$ Xiong Chen, ${ }^{\#}$ Hui-Jun Zhang, ${ }^{\dagger}$ \\ Jianbin Lin*,†,\& and Yun-Bao Jiang ${ }^{\dagger, \S}$ \\ ${ }^{\dagger}$ Department of Chemistry, College of Chemistry and Chemical Engineering, Xiamen University, Xiamen 361005, P. R. China \\ $\$$ School of Chemistry and Chemical Engineering, Beijing Institute of Technology, Beijing 100081, P. R. China \\ \# State Key Laboratory of Photocatalysis on Energy and Environment, and Key Laboratory of Molecular Synthesis and Function Discovery, \\ College of Chemistry, Fuzhou University, Fuzhou 350116, P. R. China

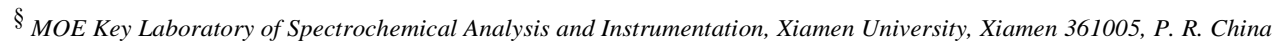

\section{General methods and materials}

General methods

${ }^{1} \mathrm{H}$ NMR and ${ }^{13} \mathrm{C}$ NMR spectra were recorded at room temperature on a Bruker AV500 NMR spectrometer. Solid-state ${ }^{13} \mathrm{C}$ NMR spectra were recorded on a Bruker $400 \mathrm{MHz}$ Avance III SS-NMR spectrometer with a CPMAS probe. Chemical shifts are given in ppm relative to tetramethylsilane. High resolution mass spectra were recorded on an FT-MS instrument using the ESI technique. SEM measurements were carried out on Hitachi S-4800. Fourier transform infrared spectra were recorded as KBr-pellet on a Nicolet 380 FT-IR spectrometer. Powder X-ray diffraction (PXRD) data were collected at $35 \mathrm{kV}, 15 \mathrm{~mA}$ on an Ultima IV XRD diffractometer using $\mathrm{Cu}-\mathrm{K} \alpha$ radiation $(\lambda=1.5418$ $\AA$ ) at room temperature. Elemental analyses were carried out on an Elementar Vario EL III Elemental Analyzer. $\mathrm{N}_{2}$ adsorption-desorption analyses were carried out at $77 \mathrm{~K}$ using a Micromeritics ASAP 2460 Surface Area and Porosity Analyzer. SEM measurements were carried out on Hitachi S-4800. Thermogravimetry analysis (TGA) was performed on a NETZSCH TG209F1 instrument in an atmosphere of $\mathrm{N}_{2}$ at a heating rate of $10{ }^{\circ} \mathrm{C} \mathrm{min}^{-1}$. Diffuse reflectance UV-Vis absorption spectra and Fluorescence spectra were obtained on Varian Cary 5000 UV-Vis spectrophotometer and Hitachi F-4500, respectively.

Materials

Deuterated solvents were purchased from Cambridge Isotope Laboratories. The other materials and solvents were commercially available and used without further purification.

The structure models of the COFs were carried out using Materials Studio 2017R2 (Accelrys). For each structure, we applied the Geometry optimization to optimize the bond length and angles subject to minimize the structural energy with the Force-field calculations using the universal force field (UFF) and charges using Qeq. 


\section{Procedures and Characterization Data}
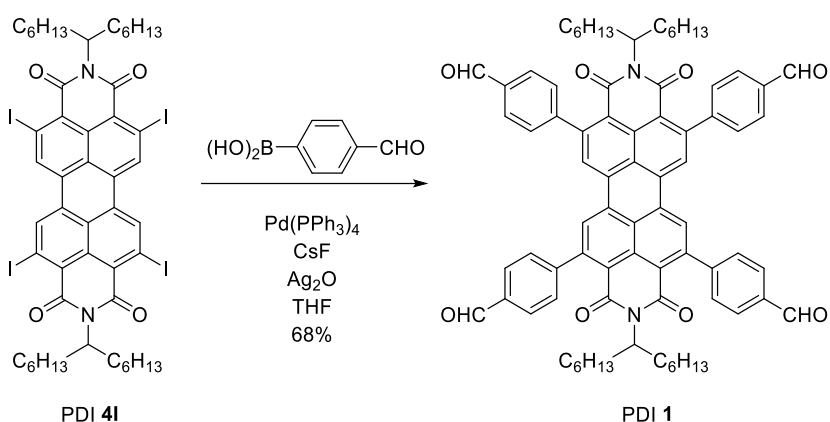

Scheme S1. Chemical synthesis of the PDI derivative PDI 1.

A mixture of PDI 4I (0.5 mmol, $645 \mathrm{mg})$, 4-Formylphenylboronic acid (2.4 mmol, $360 \mathrm{mg})$, $\mathrm{Pd}\left(\mathrm{PPh}_{3}\right)_{4}(0.1 \mathrm{mmol}, 116 \mathrm{mg}), \mathrm{CsF}(3.0 \mathrm{mmol}, 456 \mathrm{mg}), \mathrm{Ag}_{2} \mathrm{O}(2.2 \mathrm{mmol}, 273 \mathrm{mg})$ and dry THF as solvent $(10 \mathrm{~mL})$ was intensively stirred under argon at $80^{\circ} \mathrm{C}$ for 48 hours. Then, the mixture was extracted with DCM, washed with water, dried with anhydrous $\mathrm{Na}_{2} \mathrm{SO}_{4}$, filtered, and concentrated under reduced pressure. The residue was purified by silica gel column chromatography using DCM/PE (10/1) as eluent to afford PDI 1 (399 mg, 68\%).

${ }^{1} \mathrm{H}$ NMR $\left(500 \mathrm{MHz}, \mathrm{CDCl}_{3}\right) \delta=10.12(\mathrm{~s}, 4 \mathrm{H}), 8.39$ (s, 4H), 8.02 (d, J=8.0, 8H), 7.59 (d, J=8.0, $8 \mathrm{H}), 4.92-4.86(\mathrm{~m}, 4 \mathrm{H}), 2.01(\mathrm{~m}, 4 \mathrm{H}), 1.65(\mathrm{~m}, 8 \mathrm{H}), 1.26(\mathrm{~m}, 32 \mathrm{H}), 0.87(\mathrm{t}, J=7.0,12 \mathrm{H}) .{ }^{13} \mathrm{C}$ NMR $\left(126 \mathrm{MHz}, \mathrm{CDCl}_{3}\right) \delta=191.6,163.2,148.2,146.7,135.7,132.7,131.0,129.9,128.6,126.9,125.9$, 121.6, 55.1, 31.9, 31.8, 29.1, 26.9, 22.6, 14.1. HR-MS (APCI) m/z: $[\mathrm{M}+\mathrm{K}]^{+}$Calcd for $\mathrm{C}_{78} \mathrm{H}_{78} \mathrm{~N}_{2} \mathrm{O}_{8} \mathrm{~K}^{+}$1210.5858; Found 1210.58346 .

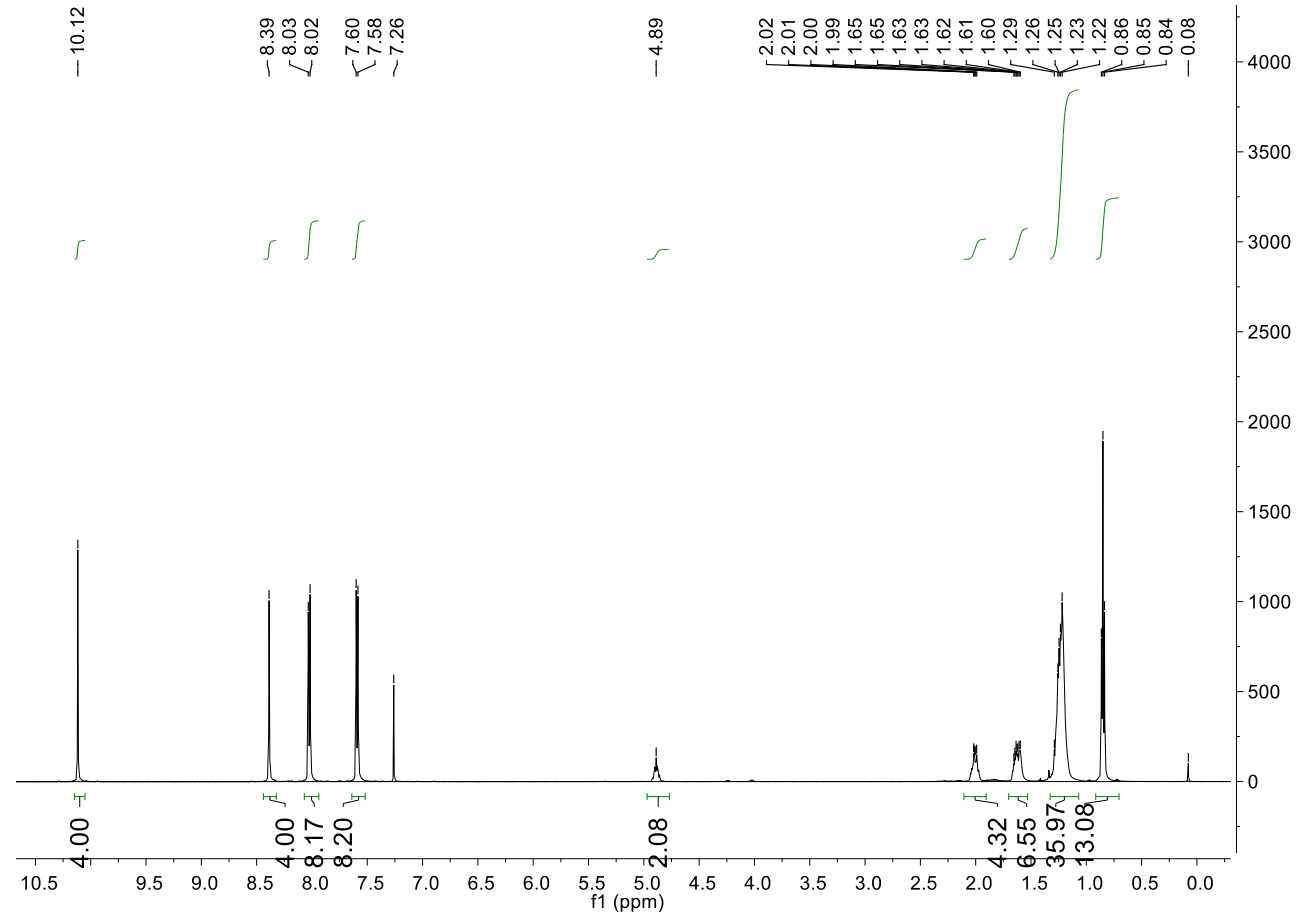



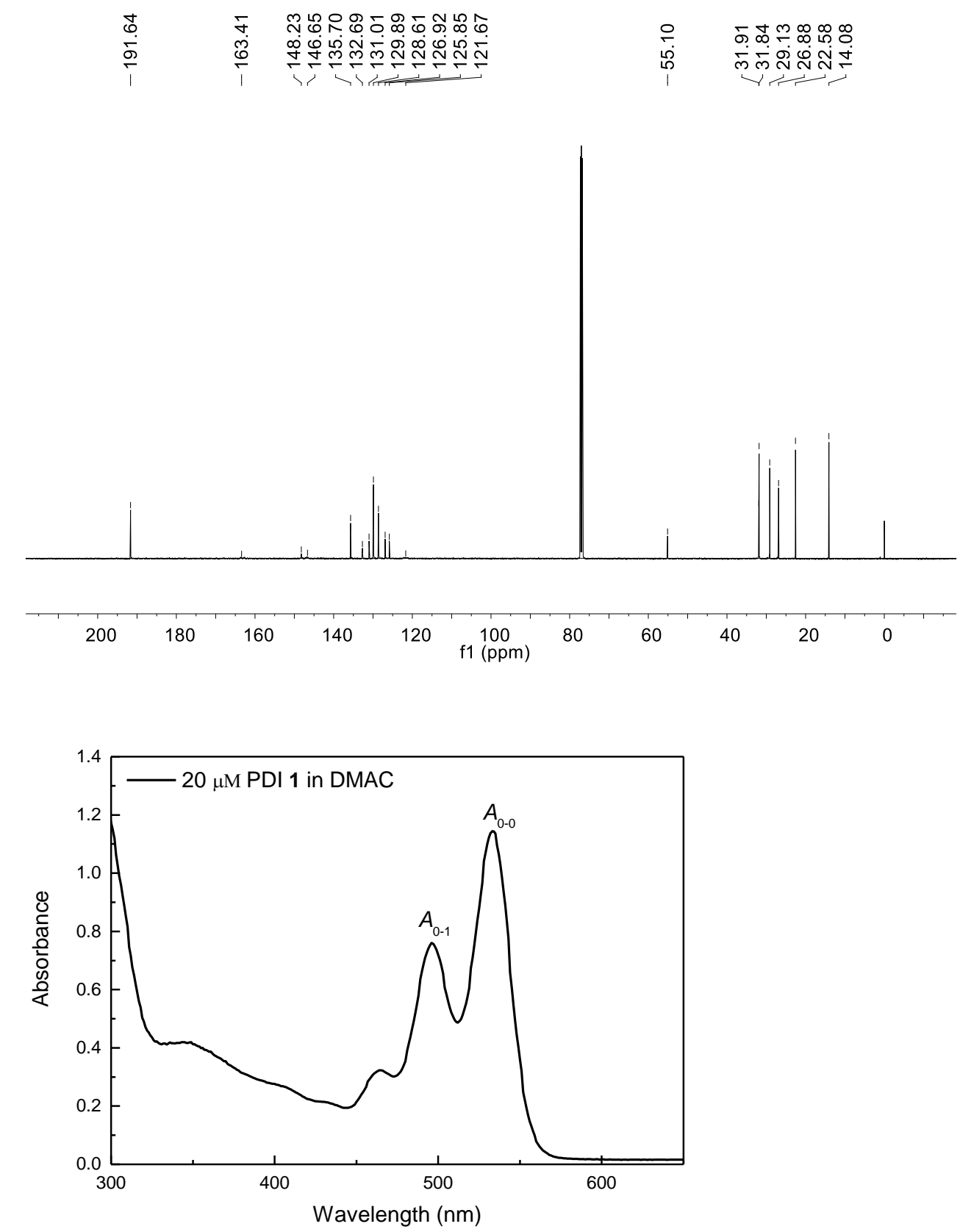

Figure S1. UV-vis absorption spectra of PDI 1 in DMAC (20 $\mu \mathrm{M})$. 


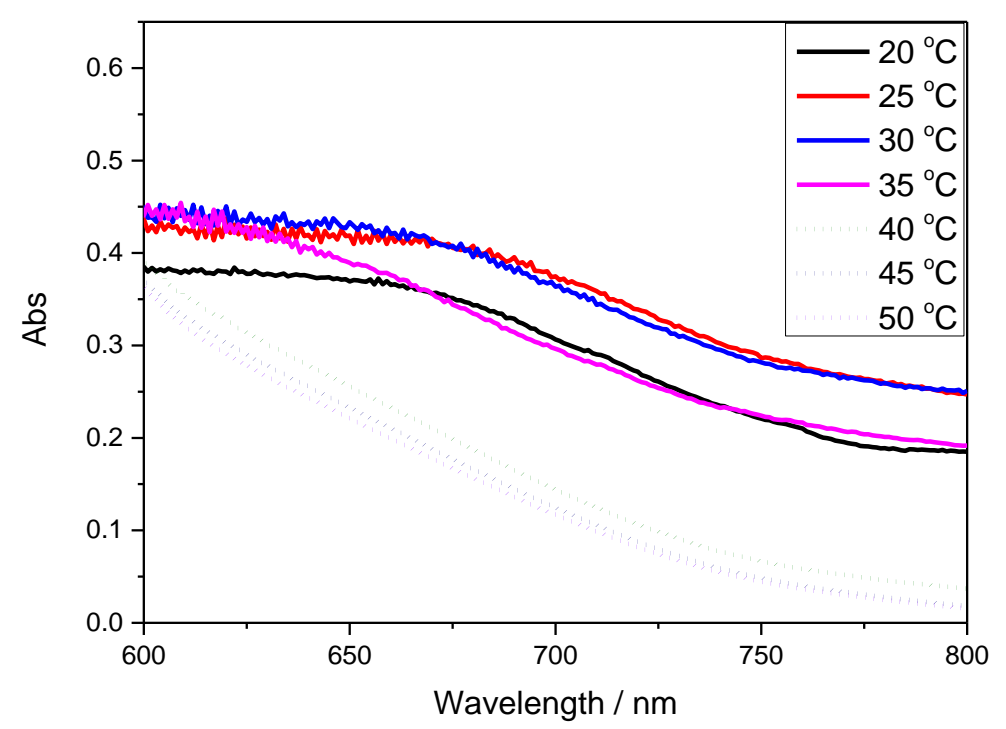

Figure S2. Partial UV-vis spectra highlighting the CT absorption changes following a series of heating of PDI 1/ perylene 3 mixture in 1:1 ratio (2.5 mM in DMAC).

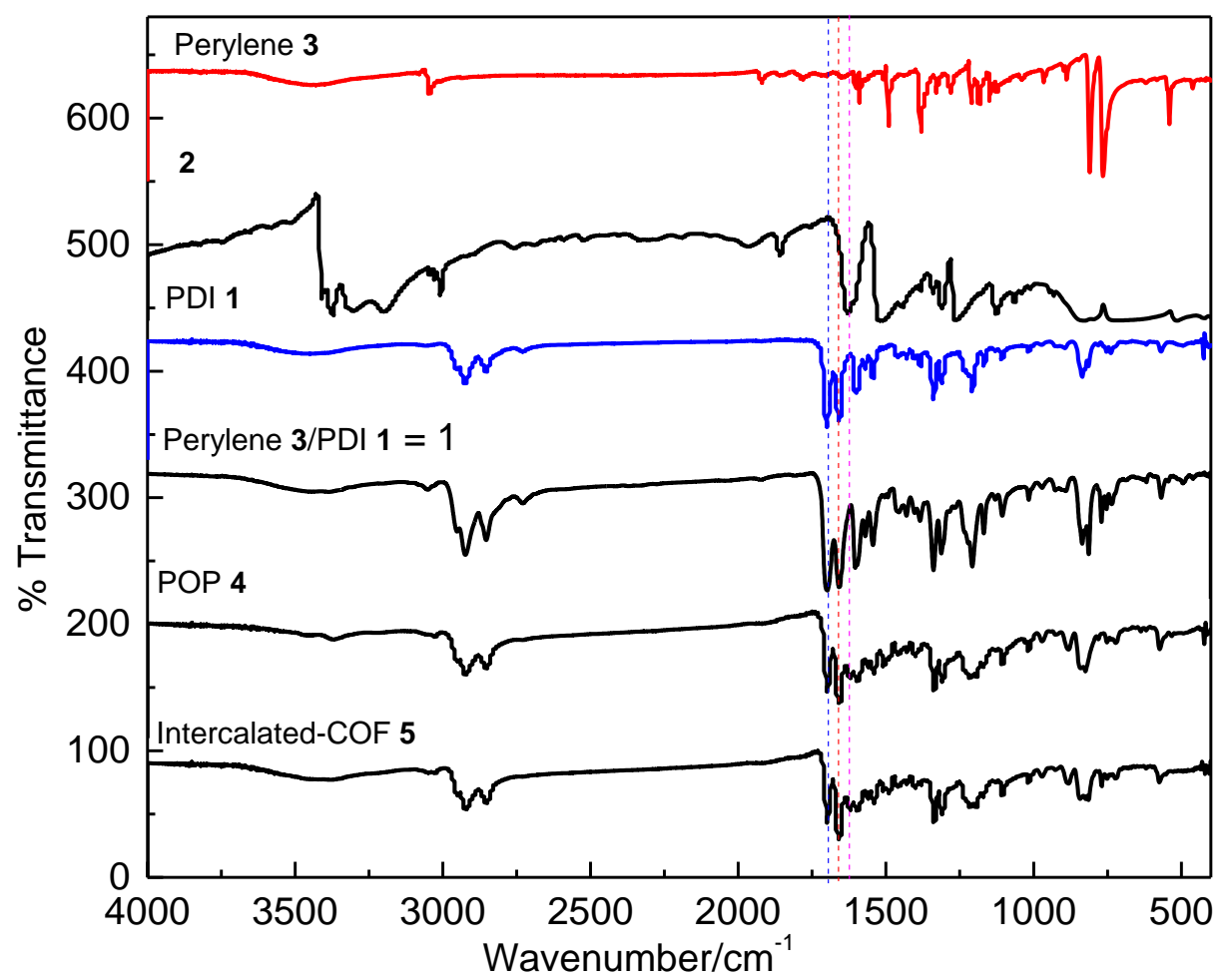

Figure S3. FT-IR spectra of Perylene 3, 2, PDI 1, PDI 1/Perylene 2 mixture in 1:1 ratio, POP 4, and Intercalated-COF $\mathbf{5}$. 
a)

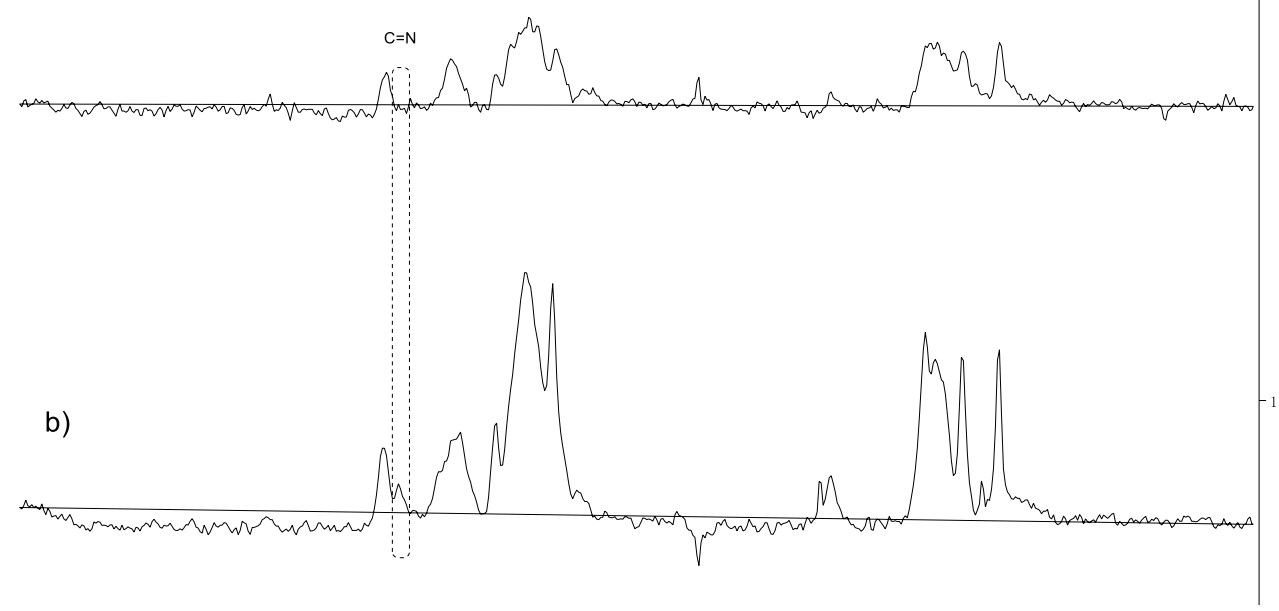

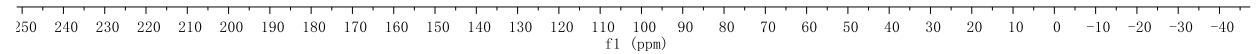

Figure S4. Solid-state CP/MAS ${ }^{13} \mathrm{C}$ NMR spectrum of a) POP 4 and b) Intercalated-COF 5.

\begin{tabular}{|l|l|l|l|l|}
\hline Compound & & N\% & C\% & H\% \\
\hline \multirow{2}{*}{ POP 4 } & Calculated: & 6.39 & 82.16 & 6.59 \\
\cline { 2 - 5 } & Found: & 5.30 & 74.57 & 6.26 \\
\hline \multirow{2}{*}{ Intercalated-COF 5 5} & Calculated: & 5.36 & 84.26 & 6.81 \\
\cline { 2 - 5 } & Found: & 5.38 & 83.17 & 6.39 \\
\hline
\end{tabular}

Table S1. Elemental analysis of POP $\mathbf{4}$ and Intercalated-COF 5.
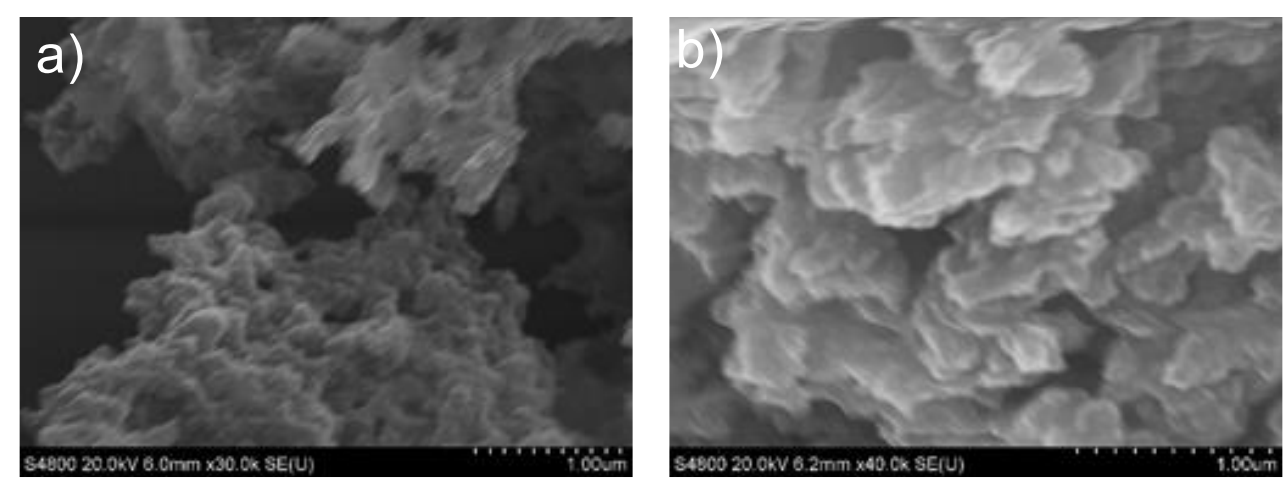

Figure S5. FESEM images of a) POP 4 and b) Intercalated-COF 5. 


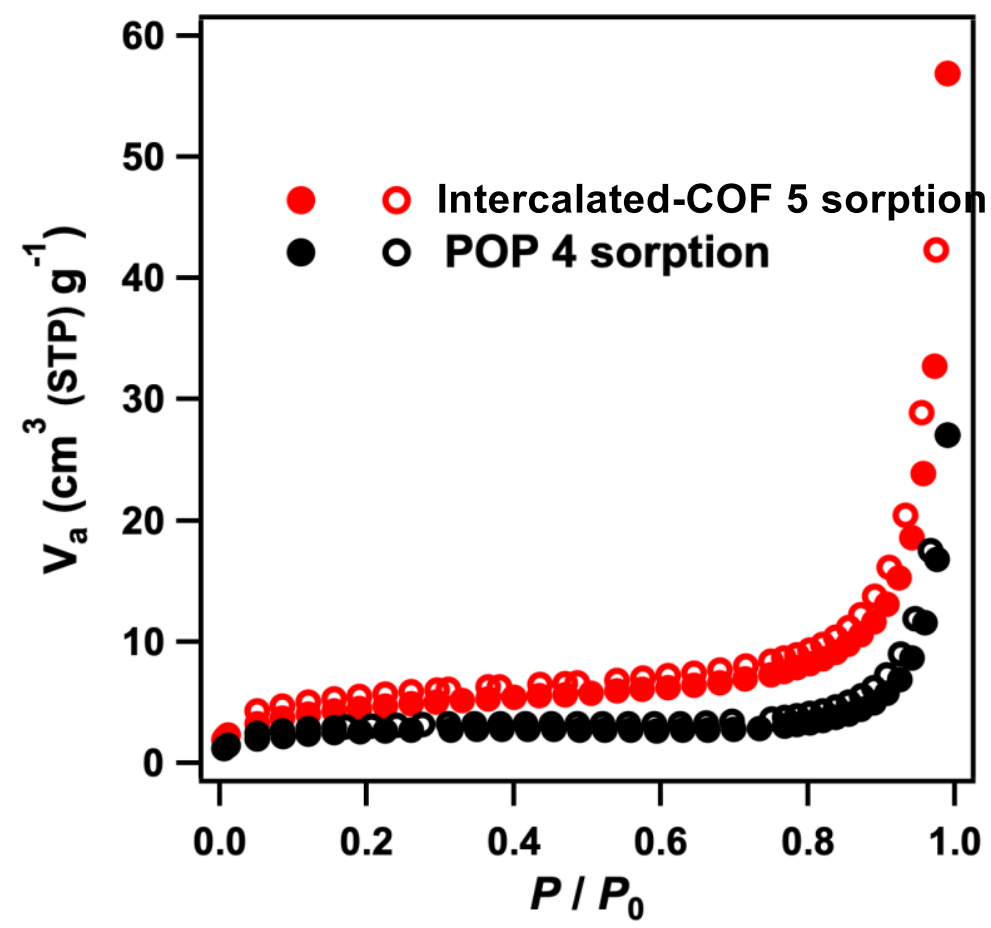

Figure S6. Gas absorption. Nitrogen sorption curves of Intercalated-COF 5 and POP-4. (filled circles: adsorption, open circles: desorption, $77 \mathrm{~K}$ ).
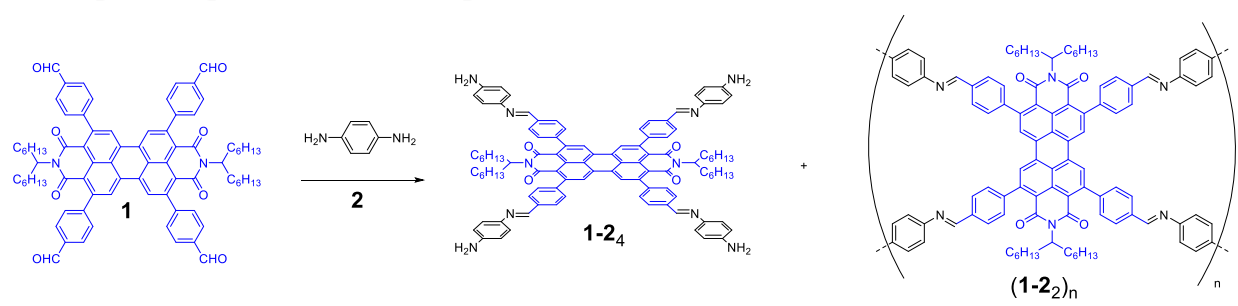

$$
\frac{+}{1}
$$
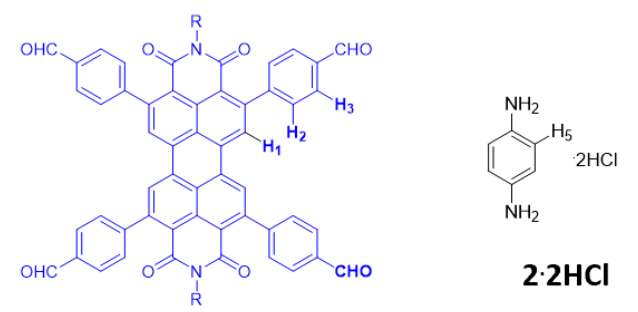

$2 \cdot 2 \mathrm{HCl}$

1

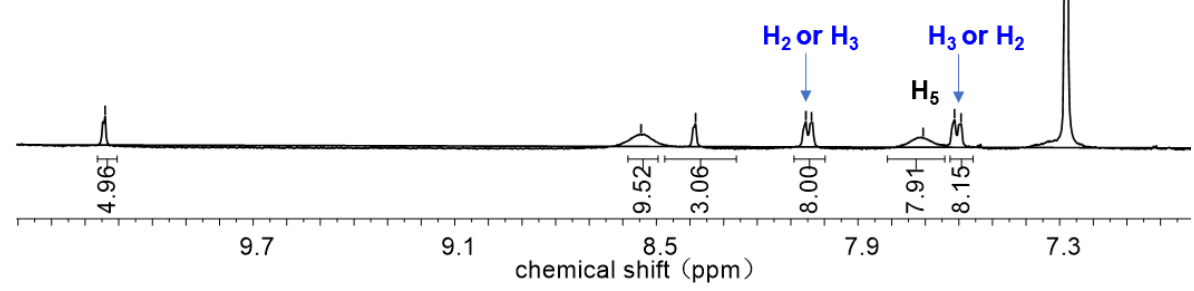

Figure S7. NMR analysis for the POP 4 after hydrochloric acid etch in DMSO-d 6 . 


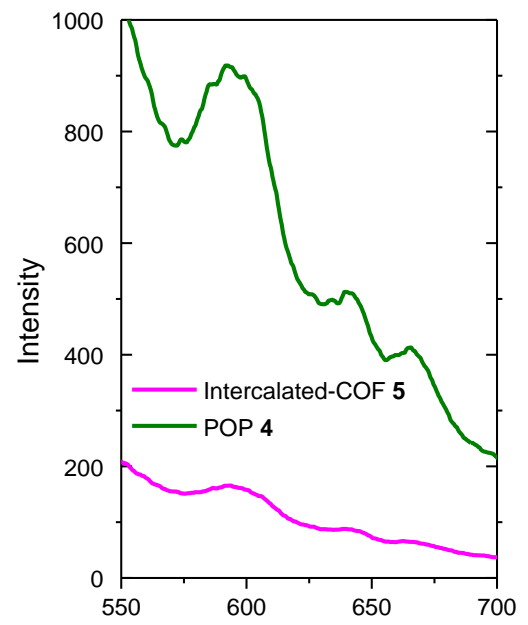

Figure S8. Fluorescence spectra of POP 4 and Intercalated-COF 5 in the solid state $\left(\lambda_{\mathrm{ex}}=496 \mathrm{~nm}\right)$.

Fractional atomic coordinates for the unit cell of Intercalated-COF 5 with AA stacking.

Unit cell parameters

$$
\begin{array}{lccccrl}
\text { a: } & 23.83480 & \text { b: } & 23.58030 & \text { c: } & 7.03480 & \text { A } \\
\text { alpha: } & 87.3564 & \text { beta: } & 83.1717 & \text { gamma: } & 120.0005 & \text { deg }
\end{array}
$$

Unit cell volume: $\quad 3374.0493 \mathrm{~A}^{\wedge} 3$

Estimated density: $\quad 771.665 \mathrm{~kg} / \mathrm{m}^{\wedge} 3$

$0.7717 \mathrm{~g} / \mathrm{cm}^{\wedge} 3$

0.0646 atoms $/ \mathrm{A}^{\wedge} 3$

Calculated formula: C $110.00 \mathrm{H} 98.00 \mathrm{~N} 6.00 \mathrm{O} 4.00$

Spacegroup symbol: $\quad * * *$ undefined $* * *$

Lattice type: $\quad P$

General Equivalent Positions:

\begin{tabular}{ccccc} 
& & $+\mathrm{x}$ & $+\mathrm{y}$ & $+\mathrm{z}$ \\
& \multicolumn{4}{c}{ Fractional Coordinates } \\
& $\mathrm{x}$ & $\mathrm{y}$ & $\mathrm{z}$ & \\
& & & \\
C1 & 0.43652 & 0.48173 & 0.81551 & \\
$\mathrm{C} 2$ & 0.44541 & 0.42835 & 0.77494 & \\
C3 & 0.50916 & 0.43957 & 0.71465 & \\
C4 & 0.56341 & 0.50551 & 0.67334 \\
C5 & 0.55417 & 0.5589 & 0.71283
\end{tabular}




\begin{tabular}{|c|c|c|c|}
\hline & & & \\
\hline C7 & 37211 & 6923 & 68 \\
\hline & 3178 & .40555 & 88457 \\
\hline & 32657 & 0.3515 & .85758 \\
\hline & 2 & & \\
\hline & 39854 & & 827 \\
\hline 12 & 265 & 51 & 836 \\
\hline 12 & 69 & & 458 \\
\hline 14 & 44 & & \\
\hline 5 & 348 & 11 & 05 \\
\hline 6 & 919 & & 644 \\
\hline 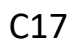 & 567 & & \\
\hline 18 & 74 & 0.5 & \\
\hline 19 & 0.67947 & 0.58482 & 0.55131 \\
\hline 0 & 66943 & 0.6 & \\
\hline & & & \\
\hline 22 & 89 & 5 & 944 \\
\hline 23 & 0.74539 & 0.59381 & 0.49853 \\
\hline 4 & .47546 & 0.2 & 636 \\
\hline & & & \\
\hline 26 & 0.17667 & 0.42758 & 0.07497 \\
\hline 27 & 1459 & 0.4 & 185 \\
\hline 8 & 9 & & \\
\hline & 1 & & \\
\hline 30 & 0.43838 & 0.2 & 0.91123 \\
\hline 31 & 0.46047 & 0.17307 & 0.97484 \\
\hline 2 & 51984 & 97 & 356 \\
\hline & 1 & & \\
\hline 34 & 5317 & 0.2 & 0.65368 \\
\hline 35 & 19 & & 181 \\
\hline & 7 & & \\
\hline & 3 & 0.8 & 21 \\
\hline 38 & 18 & & 466 \\
\hline 39 & 47198 & & 0.95234 \\
\hline & 0.76076 & 0.57 & \\
\hline 1 & 81912 & 0.56907 & 0.30073 \\
\hline 42 & 86182 & 0.59021 & 0.4365 \\
\hline & & & \\
\hline 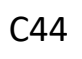 & .79015 & 0.61905 & 0.62765 \\
\hline C45 & 27078 & 0.28 & 0.89866 \\
\hline & 33886 & 43 & 0.75155 \\
\hline & 72143 & 0.70796 & 0.50467 \\
\hline C50 & 0.65138 & 0.74664 & 0.62948 \\
\hline C55 & 0.76638 & 0.82887 & 0.46957 \\
\hline
\end{tabular}




\begin{tabular}{|c|c|c|c|}
\hline 56 & 7 & 9 & \\
\hline 57 & 09867 & 0.44077 & 0.90089 \\
\hline 8 & 4711 & 34 & 46 \\
\hline & 465 & & \\
\hline & & & \\
\hline 61 & 02868 & 7993 & 0.51857 \\
\hline c62 & 05002 & 0.48445 & 0.69679 \\
\hline 63 & 03795 & 78 & \\
\hline$\theta^{2}$ & & & \\
\hline C65 & 97988 & 16 & 0.60709 \\
\hline 68 & 49762 & 0.02002 & 33 \\
\hline 9 & 33 & 0.9 & \\
\hline 70 & 7 & 2 & 287 \\
\hline 71 & 372 & 0.0 & 957 \\
\hline 72 & 6 & 0.0 & \\
\hline 3 & 2 & 0.0 & \\
\hline 76 & 21848 & 1634 & 0.85666 \\
\hline 77 & 74768 & 0.86827 & 262 \\
\hline 78 & 5 & & \\
\hline 79 & 79057 & 0.9 & 704 \\
\hline 80 & 85001 & 0.05 & 56 \\
\hline 81 & 3 & 0.1 & .76 \\
\hline 32 & & & \\
\hline 83 & 79342 & & \\
\hline C84 & 81622 & 0.82234 & 0.76436 \\
\hline 85 & 86709 & 0.86 & 84 \\
\hline 86 & 88486 & & \\
\hline $\mathrm{C} 87$ & 1 & 0.86 & \\
\hline $\mathrm{C} 88$ & 94801 & 0.82176 & 0.26845 \\
\hline C89 & 1 & 0.1 & 07 \\
\hline 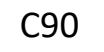 & & & \\
\hline 91 & 55 & 0.16 & 051 \\
\hline C92 & 10595 & 0.21401 & \\
\hline 93 & 7 & 52 & 48 \\
\hline 4 & 9 & 0.2 & \\
\hline 95 & 5 & 8 & 158 \\
\hline 96 & 16641 & 0.04402 & 0.02023 \\
\hline$x_{0}$ & 22 & 0.98 & 0.96921 \\
\hline C98 & 16987 & 0.97383 & 0.75317 \\
\hline 99 & 35 & 0.9 & 541 \\
\hline 0 & 0619 & 0.86917 & 241 \\
\hline C101 & 42269 & 0.4 & 0.28974 \\
\hline C102 & 0.467 & & 0.24 \\
\hline C103 & 0.53599 & 0.44711 & 0.18837 \\
\hline
\end{tabular}




\begin{tabular}{|c|c|c|c|}
\hline 104 & .55911 & & \\
\hline 05 & 8 & 58 & \\
\hline & 5 & 18 & \\
\hline & & & \\
\hline & & & \\
\hline 09 & & & 52 \\
\hline 10 & 44431 & 0.3 & 2511 \\
\hline & & & \\
\hline & & & \\
\hline 13 & 57907 & 41 & 0. \\
\hline 4 & 9 & & \\
\hline & & & \\
\hline 16 & & & \\
\hline 17 & 15 & 18 & 12 \\
\hline 18 & 38 & & \\
\hline & & & \\
\hline 20 & 774 & & 0.2 \\
\hline 21 & & 0.5 & 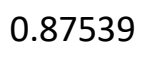 \\
\hline 2 & & & \\
\hline 23 & & 0.5 & 0.9 \\
\hline 24 & 96 & & \\
\hline 25 & & & \\
\hline 126 & & & \\
\hline 27 & & 0.3 & \\
\hline 28 & 9 & & 39 \\
\hline 2 & & & \\
\hline 30 & & & \\
\hline 31 & 3 & $0 ?$ & \\
\hline 32 & & 3 & \\
\hline 33 & & & \\
\hline & & & \\
\hline 135 & 3 & 7 & 61 \\
\hline H136 & 43226 & 2 & 0.8 \\
\hline & & & \\
\hline 128 & 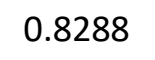 & 7 & 576 \\
\hline 139 & 65 & 99 & 283 \\
\hline 40 & & & 52 \\
\hline & & & \\
\hline H142 & 6 & 18 & 516 \\
\hline H143 & 0.07839 & 0.45093 & 0.0318 \\
\hline & 8238 & & . \\
\hline B & .92727 & 0.55888 & .2932 \\
\hline & & & 0.3373 \\
\hline 147 & 0.03883 & 0.45207 & $0.416 c$ \\
\hline
\end{tabular}




\begin{tabular}{|c|c|c|c|}
\hline & & & \\
\hline 19 & 5 & & \\
\hline & & & \\
\hline 51 & 46541 & .92186 & (1000 \\
\hline & & & \\
\hline & & & \\
\hline 54 & 6 & & 17 \\
\hline & & & \\
\hline & & & \\
\hline 57 & & & \\
\hline 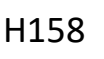 & & & \\
\hline & & & \\
\hline 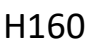 & & & \\
\hline 61 & 69 & 0.0 & 85 \\
\hline 62 & & & \\
\hline & & & \\
\hline 164 & 7 & & \\
\hline 65 & 6 & 0.1 & 36 \\
\hline 66 & & & \\
\hline & & & \\
\hline 68 & 91 & 0.8 & 06 \\
\hline 65 & & & 2 \\
\hline & & & \\
\hline & & & \\
\hline 172 & 32 & & \\
\hline 173 & 3 & 0.9 & 2 \\
\hline 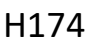 & 2 & & \\
\hline 175 & & & \\
\hline 76 & & & \\
\hline 177 & & & \\
\hline & & & \\
\hline & 4 & & \\
\hline $\mathrm{H} 18$ & & & \\
\hline & & & \\
\hline & & & \\
\hline H183 & 19669 & 6 & 0.5956 \\
\hline - & & & 0 \\
\hline & & & \\
\hline & 10 & 5 & 2110 \\
\hline 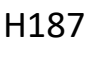 & 8 & 0.26 & 094 \\
\hline & & & 2 \\
\hline & & 0.14345 & \\
\hline & 0.08768 & 0.16921 & 0.0981 \\
\hline $\mathrm{H} 191$ & 0.03618 & 0.26226 & 0.2125 \\
\hline
\end{tabular}




$\begin{array}{cccc}\mathrm{H} 192 & 0.02123 & 0.21913 & 0.00152 \\ \mathrm{H} 193 & 0.10384 & 0.27977 & 0.02673 \\ \mathrm{H} 194 & 0.25957 & 0.1288 & 0.06096 \\ \mathrm{H} 195 & 0.26301 & 0.10198 & 0.83166 \\ \mathrm{H} 196 & 0.12298 & 0.04199 & 0.97043 \\ \mathrm{H} 197 & 0.15679 & 0.04125 & 0.18028 \\ \mathrm{H} 198 & 0.12856 & 0.9382 & 0.05831 \\ \mathrm{H} 199 & 0.2158 & 0.98751 & 0.01085 \\ \mathrm{H} 200 & 0.19024 & 0.94143 & 0.71769 \\ \mathrm{H} 201 & 0.20369 & 0.02159 & 0.66164 \\ \mathrm{H} 202 & 0.10709 & 0.957 & 0.53686 \\ \mathrm{H} 203 & 0.07374 & 0.96635 & 0.76609 \\ \mathrm{H} 204 & 0.0871 & 0.84647 & 0.66191 \\ \mathrm{H} 205 & 0.01258 & 0.84931 & 0.70031 \\ \mathrm{H} 206 & 0.0551 & 0.85399 & 0.89904 \\ \mathrm{H} 207 & 0.3176 & 0.38923 & 0.34447 \\ \mathrm{H} 208 & 0.28041 & 0.27399 & 0.33479 \\ \mathrm{H} 209 & 0.3584 & 0.235 & 0.2918 \\ \mathrm{H} 210 & 0.47147 & 0.25821 & 0.24966 \\ \mathrm{H} 211 & 0.58925 & 0.33684 & 0.15074 \\ \mathrm{H} 212 & 0.63159 & 0.45415 & 0.11769 \\ \mathrm{H} 213 & 0.51835 & 0.67692 & 0.37785 \\ \mathrm{H} 214 & 0.4002 & 0.59856 & 0.46748 \\ \mathrm{H} 215 & 0.35424 & 0.48335 & 0.43085 \\ \mathrm{H} 216 & 0.65841 & 0.55187 & 0.02554 \\ \mathrm{H} 217 & 0.70029 & 0.66613 & 0.07447 \\ \mathrm{H} 218 & 0.62849 & 0.70034 & 0.24572 \\ \mathrm{~N} 46 & 0.27757 & 0.23065 & 0.8379 \\ \mathrm{~N} 49 & 0.71113 & 0.75977 & 0.53167 \\ \mathrm{~N} 66 & 0.93802 & 0.57182 & 0.57974 \\ \mathrm{~N} 67 & 0.08713 & 0.45387 & 0.73353 \\ \mathrm{~N} 74 & 0.52631 & 0.11595 & 0.14266 \\ \mathrm{~N} 75 & 0.56016 & 0.90654 & 0.28077 \\ \mathrm{O} 51 & 0.2184 & 0.27032 & 0.98818 \\ & 0.3446 & 0.20349 & 0.66509 \\ \mathrm{O} 53 & 0.64048 & 0.79148 & 0.64566 \\ & & & \\ \mathrm{H} 1345 & 0.7191 & 0.41394\end{array}$

Fractional atomic coordinates for the unit cell of $(\mathbf{1 - 2})_{\mathrm{n}} \mathrm{COF}$ with AA stacking.

Unit cell parameters

$$
\begin{gathered}
\text { a: } 23.62450 \quad \text { b: } \quad 24.89220 \quad \text { c: } \\
\text { S12 }
\end{gathered}
$$


alpha: 90.1844 beta: 97.5922 gamma: 120.0619 deg

Unit cell volume: $\quad 2252.3003 \mathrm{~A}^{\wedge} 3$

Estimated density: $\quad 969.980 \mathrm{~kg} / \mathrm{m}^{\wedge} 3$

$0.9700 \mathrm{~g} / \mathrm{cm}^{\wedge} 3$

0.0826 atoms $/ \mathrm{A}^{\wedge} 3$

Calculated formula: C $90.00 \mathrm{H} 86.00 \mathrm{~N} 6.00 \mathrm{O} 4.00$

Spacegroup symbol: $\quad * * *$ undefined $* * *$

Lattice type: $\quad \mathrm{P}$

General Equivalent Positions:

$+\mathrm{x}$

$+y$

Fractional coordinates

$\begin{array}{cccc}\text { Label } & x & y & z \\ \text { C1 } & 0.44111 & 0.50227 & 0.67799 \\ \text { C2 } & 0.43989 & 0.44631 & 0.74106 \\ \text { C3 } & 0.49362 & 0.43909 & 0.69329 \\ \text { C4 } & 0.54614 & 0.4869 & 0.55735 \\ \text { C5 } & 0.54906 & 0.54474 & 0.51926 \\ \text { C6 } & 0.49849 & 0.55349 & 0.59819 \\ \text { C7 } & 0.38325 & 0.50444 & 0.6771 \\ \text { C8 } & 0.32572 & 0.45414 & 0.74704 \\ \text { C9 } & 0.32509 & 0.39912 & 0.83184 \\ \text { C10 } & 0.3834 & 0.39659 & 0.84164 \\ \text { C11 } & 0.38448 & 0.34372 & 0.94285 \\ \text { C12 } & 0.43965 & 0.33737 & 0.90961 \\ \text { C13 } & 0.49165 & 0.38365 & 0.77657 \\ \text { C14 } & 0.60234 & 0.59405 & 0.39854 \\ \text { C15 } & 0.60957 & 0.65387 & 0.3949 \\ \text { C16 } & 0.55972 & 0.66269 & 0.48805 \\ \text { C17 } & 0.50587 & 0.61305 & 0.58902 \\ \text { C18 } & 0.59591 & 0.47928 & 0.45804 \\ \text { C19 } & 0.64635 & 0.52628 & 0.32171 \\ \text { C20 } & 0.64822 & 0.58354 & 0.28028 \\ \text { C21 } & 0.26742 & 0.46167 & 0.69353 \\ \text { C22 } & 0.55693 & 0.72056 & 0.46061 \\ \text { C23 } & 0.69754 & 0.51468 & 0.23221 \\ \text { C24 } & 0.44431 & 0.28185 & 0.99251 \\ \text { C25 } & 0.26916 & 0.51206 & 0.84457 \\ \text { C26 } & 0.22591 & 0.53249 & 0.73218 \\ \text { C27 } & 0.18024 & 0.50243 & 0.46924 \\ \text { C28 } & 0.17611 & 0.45003 & 0.32796 \\ \text { C29 } & 0.22108 & 0.43145 & 0.43383\end{array}$




\begin{tabular}{|c|c|c|c|}
\hline C30 & 0.39851 & 0.22277 & 0.84819 \\
\hline C31 & 0.40895 & 0.17261 & 0.89305 \\
\hline C32 & 0.4647 & 0.18064 & 0.08888 \\
\hline C33 & 0.50873 & 0.23889 & 0.24437 \\
\hline C34 & 0.49941 & 0.28932 & 0.19076 \\
\hline C35 & 0.59275 & 0.77058 & 0.68184 \\
\hline C36 & 0.58087 & 0.82033 & 0.68246 \\
\hline C37 & 0.53204 & 0.81991 & 0.46427 \\
\hline C38 & 0.49697 & 0.77025 & 0.24107 \\
\hline C39 & 0.50875 & 0.72068 & 0.24185 \\
\hline $\mathrm{C} 40$ & 0.67941 & 0.46062 & 0.05261 \\
\hline C41 & 0.72672 & 0.45464 & -1.07614 \\
\hline $\mathrm{C} 42$ & 0.7928 & 0.50256 & -1.02177 \\
\hline $\mathrm{C} 43$ & 0.81198 & 0.55341 & 0.18473 \\
\hline $\mathrm{C} 44$ & 0.76471 & 0.55849 & 0.31761 \\
\hline $\mathrm{C} 45$ & 0.26411 & 0.34226 & 0.8935 \\
\hline $\mathrm{C} 47$ & 0.3276 & 0.29748 & 0.0883 \\
\hline $\mathrm{C} 48$ & 0.69673 & 0.63376 & 0.1124 \\
\hline C50 & 0.67142 & 0.70619 & 0.30798 \\
\hline C55 & 0.77701 & 0.7437 & 0.06954 \\
\hline C56 & 0.51379 & 0.86833 & 0.47464 \\
\hline C57 & 0.13978 & 0.52801 & 0.33603 \\
\hline C58 & 0.4809 & 0.13076 & 0.11393 \\
\hline C59 & 0.84253 & 0.49753 & -1.16151 \\
\hline $\mathrm{C} 60$ & 0.95473 & 0.49507 & -1.35905 \\
\hline C61 & 0.00772 & 0.48508 & -1.28174 \\
\hline C62 & 0.06239 & 0.5273 & -1.0748 \\
\hline $\mathrm{C} 63$ & 0.06943 & 0.58492 & 0.01055 \\
\hline C64 & 0.01651 & 0.59472 & -1.0665 \\
\hline C65 & 0.95711 & 0.5475 & -1.22608 \\
\hline C68 & 0.43879 & -1.00755 & 0.63761 \\
\hline C69 & 0.45587 & -1.05266 & 0.58551 \\
\hline $\mathrm{C} 70$ & 0.51161 & -1.04962 & 0.76047 \\
\hline C71 & 0.54799 & -1.00239 & 0.99328 \\
\hline $\mathrm{C} 72$ & 0.53083 & 0.04271 & 0.04586 \\
\hline C73 & 0.47576 & 0.04028 & 0.86706 \\
\hline C76 & 0.21011 & 0.24195 & 0.16708 \\
\hline $\mathrm{C} 77$ & 0.78654 & 0.81006 & 0.07162 \\
\hline C78 & 0.84906 & 0.85882 & 0.95205 \\
\hline C79 & 0.8438 & 0.91622 & 0.87835 \\
\hline $\mathrm{C} 80$ & 0.90458 & 0.96349 & 0.74651 \\
\hline $\mathrm{C} 81$ & 0.89817 & 0.01975 & 0.66185 \\
\hline $\mathrm{C} 82$ & 0.95733 & 0.06519 & 0.51587 \\
\hline C83 & 0.83623 & 0.74348 & 0.26685 \\
\hline
\end{tabular}




\begin{tabular}{|c|c|c|c|}
\hline C84 & 0.88363 & 0.73756 & 0.08335 \\
\hline $\mathrm{C} 85$ & 0.94136 & 0.74019 & 0.29862 \\
\hline $\mathrm{C} 86$ & 0.99851 & 0.75218 & 0.13104 \\
\hline C87 & 0.05629 & 0.75716 & 0.35346 \\
\hline $\mathrm{C} 88$ & 0.11609 & 0.77527 & 0.19432 \\
\hline C89 & 0.14816 & 0.24901 & 0.14882 \\
\hline C90 & 0.08993 & 0.20071 & 0.29457 \\
\hline C91 & 0.0506 & 0.22933 & 0.40116 \\
\hline C92 & 0.00635 & 0.23666 & 0.14258 \\
\hline C93 & 0.96721 & 0.26249 & 0.27207 \\
\hline C94 & 0.92421 & 0.27262 & 0.01953 \\
\hline C95 & 0.19185 & 0.17827 & 0.00942 \\
\hline C96 & 0.19666 & 0.13292 & 0.22972 \\
\hline C97 & 0.18651 & 0.07399 & 0.06227 \\
\hline C98 & 0.11341 & 0.02863 & 0.9368 \\
\hline C99 & 0.10567 & 0.96979 & 0.78401 \\
\hline C100 & 0.03341 & 0.92548 & 0.64905 \\
\hline H101 & 0.37932 & 0.54312 & 0.59151 \\
\hline H102 & 0.53027 & 0.37471 & 0.7431 \\
\hline H103 & 0.47057 & 0.6229 & 0.66674 \\
\hline H104 & 0.59674 & 0.43682 & 0.48086 \\
\hline H105 & 0.30648 & 0.53747 & 0.03955 \\
\hline H106 & 0.22934 & 0.57278 & 0.84521 \\
\hline H107 & 0.14177 & 0.42673 & 0.12179 \\
\hline H108 & 0.22355 & 0.39683 & 0.30121 \\
\hline H109 & 0.35605 & 0.21627 & 0.694 \\
\hline $\mathrm{H} 110$ & 0.37458 & 0.12795 & 0.76996 \\
\hline H111 & 0.55217 & 0.24612 & 0.3953 \\
\hline $\mathrm{H} 112$ & 0.53633 & 0.33469 & 0.29598 \\
\hline $\mathrm{H} 113$ & 0.62782 & 0.76959 & 0.8596 \\
\hline H114 & 0.6078 & 0.85789 & 0.85944 \\
\hline H115 & 0.45901 & 0.76894 & 0.07118 \\
\hline H116 & 0.47871 & 0.68123 & 0.07805 \\
\hline H117 & 0.62852 & 0.42426 & 0.004 \\
\hline H118 & 0.71159 & 0.41391 & -1.22555 \\
\hline H119 & 0.86362 & 0.58866 & 0.24435 \\
\hline H120 & 0.77967 & 0.59785 & 0.47597 \\
\hline $\mathrm{H} 121$ & 0.77547 & 0.73072 & -1.17166 \\
\hline H122 & 0.48328 & 0.87058 & 0.27827 \\
\hline H123 & 0.1379 & 0.56313 & 0.47004 \\
\hline $\mathrm{H} 124$ & 0.516 & 0.13427 & 0.30533 \\
\hline $\mathrm{H} 125$ & 0.83451 & 0.45148 & -1.21224 \\
\hline H126 & 0.91023 & 0.45972 & -1.49911 \\
\hline H127 & 0.00397 & 0.44203 & -1.35946 \\
\hline
\end{tabular}




\begin{tabular}{|c|c|c|c|}
\hline $\mathrm{H} 128$ & 0.11316 & 0.61999 & 0.1556 \\
\hline H129 & 0.01814 & 0.63507 & 0.03355 \\
\hline H130 & 0.39697 & -1.00913 & 0.49685 \\
\hline H131 & 0.42529 & -1.08946 & 0.40941 \\
\hline H132 & 0.59008 & -1.00063 & 0.1326 \\
\hline H133 & 0.56121 & 0.07921 & 0.22324 \\
\hline H134 & 0.22692 & 0.2433 & 0.41332 \\
\hline H135 & 0.78931 & 0.82747 & 0.30366 \\
\hline H136 & 0.74192 & 0.80622 & 0.93197 \\
\hline H137 & 0.85481 & 0.83924 & 0.74621 \\
\hline H138 & 0.89394 & 0.87215 & 0.12089 \\
\hline H139 & 0.83909 & 0.93753 & 0.08537 \\
\hline $\mathrm{H} 140$ & 0.79832 & 0.90226 & 0.71124 \\
\hline H141 & 0.90895 & 0.94125 & 0.54188 \\
\hline H142 & 0.95042 & 0.97842 & 0.91123 \\
\hline H143 & 0.89519 & 0.04338 & 0.86627 \\
\hline H144 & 0.85157 & 0.00443 & 0.50115 \\
\hline H145 & 0.96697 & 0.04107 & 0.3378 \\
\hline H146 & 0.00265 & 0.08948 & 0.68719 \\
\hline H147 & 0.9468 & 0.09984 & 0.407 \\
\hline H148 & 0.81767 & 0.70483 & 0.41721 \\
\hline H149 & 0.86537 & 0.78654 & 0.4166 \\
\hline H150 & 0.90286 & 0.77635 & 0.93358 \\
\hline H151 & 0.85658 & 0.69254 & 0.93977 \\
\hline H152 & 0.92377 & 0.69576 & 0.40965 \\
\hline H153 & 0.9598 & 0.77805 & 0.48091 \\
\hline H154 & 0.01523 & 0.79645 & 0.01899 \\
\hline H155 & 0.9813 & 0.71353 & 0.95341 \\
\hline H156 & 0.04084 & 0.71177 & 0.45385 \\
\hline H157 & 0.0705 & 0.79306 & 0.54209 \\
\hline H158 & 0.15719 & 0.77724 & 0.35592 \\
\hline H159 & 0.13388 & 0.82189 & 0.10627 \\
\hline H160 & 0.10355 & 0.74078 & 0.00276 \\
\hline H161 & 0.12848 & 0.24678 & 0.91135 \\
\hline H162 & 0.16518 & 0.29591 & 0.25262 \\
\hline H163 & 0.10904 & 0.18461 & 0.48946 \\
\hline H164 & 0.05691 & 0.15879 & 0.13551 \\
\hline H165 & 0.08487 & 0.276 & 0.52505 \\
\hline H166 & 0.01973 & 0.19935 & 0.5651 \\
\hline H167 & 0.97165 & 0.19079 & 0.01385 \\
\hline H168 & 0.03764 & 0.2697 & 0.98699 \\
\hline H169 & 0.00203 & 0.30772 & 0.40761 \\
\hline $\mathrm{H} 170$ & 0.93517 & 0.22927 & 0.42538 \\
\hline H171 & 0.9555 & 0.30771 & 0.87147 \\
\hline
\end{tabular}




$\begin{array}{cccc}\mathrm{H} 172 & 0.89536 & 0.29045 & 0.11778 \\ \mathrm{H} 173 & 0.88867 & 0.22823 & 0.88184 \\ \mathrm{H} 174 & 0.22535 & 0.18659 & 0.84162 \\ \mathrm{H} 175 & 0.14088 & 0.15475 & 0.88638 \\ \mathrm{H} 176 & 0.15978 & 0.11996 & 0.38619 \\ \mathrm{H} 177 & 0.24687 & 0.15666 & 0.36796 \\ \mathrm{H} 178 & 0.20486 & 0.05012 & 0.22358 \\ \mathrm{H} 179 & 0.21713 & 0.08683 & 0.87694 \\ \mathrm{H} 180 & 0.09427 & 0.05085 & 0.76939 \\ \mathrm{H} 181 & 0.08324 & 0.01599 & 0.12345 \\ \mathrm{H} 182 & 0.1228 & 0.94588 & 0.9515 \\ \mathrm{H} 183 & 0.13692 & 0.98247 & 0.60108 \\ \mathrm{H} 184 & 0.0293 & 0.88529 & 0.51948 \\ \mathrm{H} 185 & 0.01441 & 0.94913 & 0.49065 \\ \mathrm{H} 186 & 0.00196 & 0.90781 & 0.83015 \\ \mathrm{~N} 46 & 0.2671 & 0.29471 & 0.04278 \\ \mathrm{~N} 49 & 0.71351 & 0.69497 & 0.16483 \\ \mathrm{~N} 66 & 0.89888 & 0.54736 & -1.17486 \\ \mathrm{~N} 67 & 0.10567 & 0.50743 & 0.06744 \\ \mathrm{~N} 74 & 0.45804 & 0.0872 & 0.89582 \\ \mathrm{~N} 75 & 0.53125 & 0.90523 & 0.71719 \\ \mathrm{O} 51 & 0.21114 & 0.33782 & 0.80185 \\ \text { O52 } & 0.33499 & 0.26197 & 0.2533 \\ \text { O53 } & 0.68484 & 0.75916 & 0.37224 \\ \mathrm{O} 54 & 0.72192 & 0.62071 & -1.07525\end{array}$

